\title{
Los enfoques de la literatura infantil relacionada con laEducación Física y el Deporte: un análisis comparativo entre Brasil y España \\ Approaches of children's literature dealing with Physical Education and Sport: a comparative analysis between Brazil and Spain
}

\author{
Rafael Guimarães Botelho \\ Instituto Federal de Educação, Ciência e Tecnologia do Rio de Janeiro
}

Resumen: El objetivo de este artículo es comparar los enfoques de los libros infantiles con temática relacionada con la Educación Física y el Deporte publicados en Brasil con los publicados en España. Para llevar a cabo la investigación, se ha utilizado el análisis de contenido. Además, se ha creado y utilizado un modelo heurístico para identificar los enfoques presentes en las obras infantiles publicadas en ambos países. Para validar el análisis, se ha empleado la fiabilidad intraobservador e interobservadores mediante la aplicación del índice kappa de Cohen. Los resultados de este análisis indican que: (a) predomina en gran medida el enfoque de los Juegos y recreación en los dos corpora de libros analizados; (b) el enfoque de los Deportes también registra una gran incidencia en las obras infantiles de los dos países; (c) el enfoque de la Historia presenta una marcada incidencia en ambos países; (d) otro enfoque significativo presente en las obras infantiles analizadas es el de la Ética; (e) el enfoque de la Salud presenta casi los mismos porcentajes tanto en un país como en otro; (f) otra de las similitudes encontradas es que ambos países no registran incidencia alguna de los enfoques de la Educación gerontológica y del Multiculturalismo; (g) el enfoque de los Estudios olímpicos presenta una importante incidencia en España, mientras que en Brasil el tema está olvidado; (h) el enfoque de la Danza en Brasil apenas tiene incidencia (solamente el 1,11\%), mientras que en España presenta una importante incidencia (un 8,33\%).

Palabras clave: literatura infantil, Educación Física, análisis de contenido, análisis comparativo, Brasil, España.

Abstract: The aim of this paper is to compare the approaches of children's books dealing with Physical Education and Sport published in Brazil with the ones published in Spain. To develop the investigation, a content analysis was utilized. Also, a heuristic model was created and utilized to identify the approaches present in the children's books published in both countries. To validate the analysis, was employed the intraobserver and interobserver reliability, applying Cohen's kappa coefficient. The results of this analysis indicate that: (a) the approach of Games and recreation presents the biggest incidence in both corpora; (b) the approach of Sports also registers a great incidence in the children's books of both countries; (c) the approach of History presents a significant incidence in both countries; (d) another significant approach present in the children's books analyzed is Ethics; (e) the approach of Health presents almost the same percentages in Brazil and in Spain; ( $f$ ) another similarity is that in both countries there is no incidence of the approaches of Gerontologic Education and Multiculturalism; (g) the approach of Olympic Studies presents an important incidence in Spain, though in Brazil it is overlooked; (h) the approach of Dance in Brazil has almost no incidence (only 1,11\%), though in Spain it is quite the opposite (incidence of $8,33 \%)$.

Keywords: children’s literature, Physical Education, content analysis, comparative analysis, Brazil, Spain.

\section{Introducción}

En las revistas científicas internacionales es posible identificar artículos que investigan la relación entre la literatura infantil y la Educación Física y el Deporte en Brasil (Botelho, 2012), en España (Botelho \& Oliveira, 2011) y en Estados Unidos (Fingon, 2011; Weiller \& Higgs, 1989).

No obstante, los artículos que realizan análisis comparativos todavía son considerados incipientes, ya que son pocos los autores que se interesan por investigar las similitudes y las diferencias entre dos o más corpora de obras infantiles en el área de Educación Física.

Dehecho, se puede afirmar que aún queda mucho por hacer cuando el asunto es conocer y comparar, en los casos de Brasil y de España, los enfoques de los libros de literatura infantil con temática relacionada con la Educación Física y el Deporte. En otras palabras, hace falta someter esos libros infantiles a diferentes análisis para que se puedan conocer sus verdaderos enfoques y los mensajes que difunden.

Estos análisis son necesarios porque permiten identificar qué mensajes subliminales y sistemas de valores subyacentes transmiten los libros infantiles, además de verificar si estas obras presentan, en sus contenidos, manifestaciones de estereotipos, prejuicios y discriminaciones positivas o negativas relacionadas con la Educación Física y el Deporte.

Como bien lo subraya Botelho (2010), «en realidad, la expresión

Fecha recepción: 23-05-13- Fecha envío revisores: 26-05-13- Fecha de aceptación: 11-07-13 Rafael Guimarães Botelho

Campus Arraial do Cabo

Rua José Pinto de Macedo, s/n, CEP: 28930-000, Prainha, Arraial do Cabo, RJ, Brasil

rafaelgbotelho@ig.com.br literatura infantil ni siquiera es mencionada en el quehacer de la mayoría de los autores y autoras del área de Educación Física» (p. 52).

Hay que recordar, pues, que el campo escolar de la Educación Física en Brasil y en España presenta discusiones e inquietudes semejantes (Pirolo \& Terra, 2003).

Por tanto, y en base a todo lo expuesto, se plantea el siguiente interrogante: ¿cuáles son las similitudes y las diferencias de los enfoques de la literatura infantil relacionada con la Educación Física y el Deporte en Brasil y en España?

Con el fin de dar respuesta al interrogante planteado, el objetivo de este artículo es comparar los enfoques de los libros infantiles con temática relacionada con la Educación Física y el Deporte publicados en Brasil con los publicados en España.

A modo de justificación, se señala que la utilización de libros de literatura infantil en el área de Educación Física puede desarrollar diferentes aspectos educativos y temas como, por ejemplo, los juegos, los Juegos Olímpicos, el ejercicio físico, la salud, la música, la danza, el ballet, algunos elementos de la expresión corporal y la dramatización, la cooperación, la educación ambiental, la educación en valores, la educación para la paz y la no-violencia en el Deporte.

\section{Materiales y Método}

\section{Objeto de estudio}

Ciento cincuenta (150) libros de literatura infantil, de los cuales:

a) noventa (90) obras publicadas originalmente en lengua portuguesa o traducidas a este idioma;

b) sesenta (60) libros publicados originalmente en lengua castellana o traducidos a este idioma. 


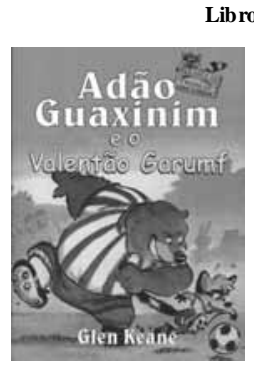

(Keane, 1995)

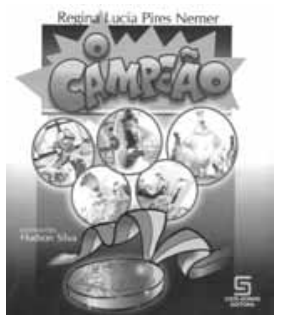

(Nemer, 2000)

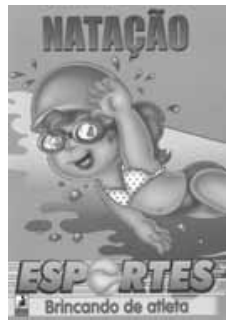

(Moraes \& Silveira, 2002)
Libros infantiles publicados en España

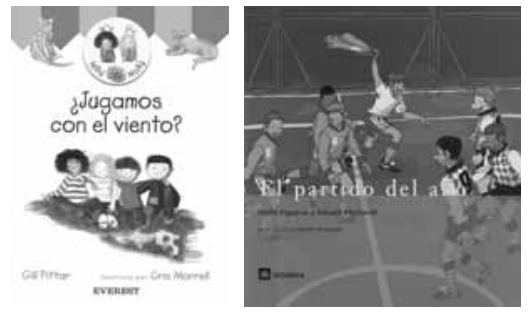

(Pittar, 2004) (Figueras Adell \& Martorell, 2007)



(Egaña, 2008)
Figura 1. Imágenes de algunas de las obras in fantiles publica das en Brasil y en España.

\section{Delimitaciones del objeto de estudio}

- Temática. Se eligieron los libros infantiles cuyos temas eran los juegos, los deportes, la recreación y el ocio, la danza, el ejercicio físico, el entrenamiento, el Olimpismo, la salud y la promoción de la salud, además de otros ejemplos específicos o relacionados directamente con el área de Educación Física y con el Deporte.

- Género literario. Obras de narrativa y de poesía.

- Temporal. Libros de literatura infantil publicados en un período de, aproximadamente, 22 años (1988-2009) en Brasil, y 35 años (1975 - 2009) en España.

- Lugar de publicación. Editoriales con sede en Brasil y en España o empresas y sociedades limitadas de traducción también con sede en Brasil y en España.

\section{Técnica de análisis}

\section{El análisis de contenido}

El análisis de contenido ya constituye una técnica de gran utilidad en las investigaciones en el área de Educación Física, y también en el campo del Deporte (Berg \& Latin, 2008; Delgado \& Del Villar, 1995; Heinemann, 2008).

En términos sencillos, «el análisis de contenido es un conjunto de técnicas de análisis de comunicaciones» (Bardin, 2002, p. 23).

Heinemann (2008) afirma que "para la investigación deportiva y para todas sus subdisciplinas existe una enorme cantidad de material objeto del análisis de contenido» (p. 165). Los «textos literarios, novelas y relatos cuyo tema es el deporte» (p. 166) son algunos de los ejemplos en los que se puede aplicar este tipo de análisis.

Al igual que Heinemann (2008), Bardin (2002) señala que una de las metas del análisis de contenido es «desenmascarar la axiología subyacente en los manuales escolares» (p. 23).

Esta investigación se desarrolló de acuerdo con las diferentes fases del análisis de contenido: 1) «El preanálisis; 2) El aprovechamiento del material; 3) El tratamiento de los resultados, la inferencia y la interpretación» (Bardin, 2002, p. 71).

\section{Definición de la variable de análisis}

Variable - Enfoque. Son los bloques que vertebran las obras de literatura infantil. Cada enfoque es una categoría. En total, son 16 categorías, que se encuentran en el modelo heurístico.
Instrumento del análisis de contenido: el modelo heurístico

El presente modelo heurístico ha sido creado para analizar libros infantiles en Brasil y España en el área de Educación Física.

Un instrumento heurístico posee un valor de descubrimiento, de invento. Su creación está relacionada con la resolución de un problema específico. Por consiguiente, este instrumento tendrá valor si, una vez aplicado, ha contribuido al progreso de un campo científico determinado (Botelho, 2010).

Por tanto, se resume el proceso que se llevó a cabo para la confección de este modelo, que comprendió las siguientes fases:

1. ${ }^{\text {- }}$ - Definición del propósito del instrumento.

2. ${ }^{\text {a }}$ - Lectura inicial de los libros infantiles y confección de un primer modelo heurístico.

3. a- Validación de contenido del modelo heurístico por un equipo de jueces (expertos).

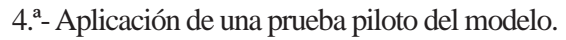

5. a- - Determinación, fundamentación y diseño del modelo heurístico definitivo.

Tras estas fases, se presenta el modelo heurístico definitivo, constituido por dieciséis categorías (enfoques).

Los enfoques del modelo heurístico tienen su origen en dos ámbitos, a saber:

- Naturaleza perpendicular. Está relacionada con los enfoques específicos, intrínsecos al área de Educación Física y al campo del Deporte. Ésta presenta un total de ocho categorías (Botelho, 2010).

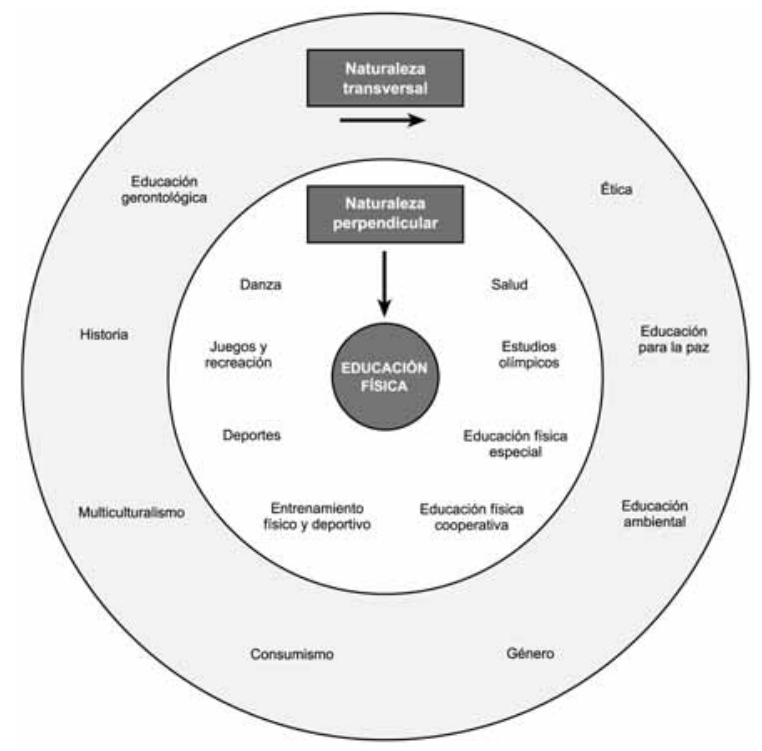

Figura 2. Modelo heurístico para la identificación de los e nfoques presentes en los libros infantiles publicados en Brasil y en España con temática rela cionada con la Educación Física y el Deporte (Botelho, 2010, p. 306).

- Naturaleza transversal. Está relacionada con los enfoques extrínsecos a la Educación Física y al Deporte, y sus categorías se originan en diferentes áreas, como la Educación, la Historia, la Sociología y la Filosofía, pero, al mismo tiempo, ejercen influencia en el área de Educación Física. Ésta también presenta un total de ocho categorías (Botelho, 2010).

A continuación, se presenta la definición de las categorías del modelo heurístico (Botelho, 2010).

\section{Naturaleza perpendicular}

Salud. Desde siempre se ha asociado la salud con el área de Educación Física. En esta categoría se incluyen los libros infantiles que presentan en su narrativa los tópicos siguientes: la educación para la salud, la promoción de la salud, la higiene corporal, la actividad y la salud, y la práctica deportiva y la seguridad (por ejemplo, la prevención de accidentes a través de la educación vial). 
Estudios olímpicos. Esta categoría está vinculada con las manifestaciones relacionadas con los Juegos Olímpicos y el Movimiento Olímpico. Un libro infantil cuya narrativa aborda temas como la filatelia, los símbolos (antorcha, mascotas, medallas, anillos, etc.), las ceremonias de apertura y clausura, la Carta Olímpica u otros será incluido en esta categoría.

Educación física especial. Esta categoría tiene relación con las historias sobre los personajes que presentan dificultades de aprendizaje a causa de alguna discapacidad o, dicho de otra forma, que tienen necesidades educativas especiales. Una narrativa que discuta, por ejemplo, un deporte adaptado a las personas con problemas intelectuales, sensoriales y físico-motrices será incluida en esta categoría.

Educación física cooperativa. Esta clase de Educación Física tiene como finalidad desarrollar actividades, ejercicios y juegos colectivos no competitivos en los que todas las personas buscan un mismo objetivo, independientemente de que desempeñen el mismo rol o funciones complementarias. Además, no hay oposición entre las acciones de los participantes. Las palabras clave de esta categoría son cooperación, ayuda y unión. El libro infantil que utilice esta idea para narrar una historia formará parte de esta categoría. En cambio, no se encuadra en esta categoría una historia o texto en el que haya un vencedor y perdedores.

Entrenamiento físico y deportivo. Será clasificado en esta categoría cualquier libro infantil que presente en su texto la idea de la práctica del ejercicio físico, del entrenamiento para un deporte, y, también, del acondicionamiento físico general con vistas a la preparación y la superación física y psicológica de una persona o un atleta en una actividad física determinada. La persistencia, la repetición y la resistencia para lograr un objetivo determinado son ejemplos de esta categoría.

Deportes. Esta categoría incluye cualquier manifestación relacionada con un deporte determinado. Los libros infantiles que discuten un deporte en concreto, que presentan las competiciones deportivas o que describen el concepto de deporte deberán ser clasificados en esta categoría.

Juegos y recreación. Esta categoría aborda un abanico de manifestaciones como los juegos tradicionales, autóctonos y folclóricos, los juguetes, los pasatiempos y el ocio. Será clasificado en esta categoría un libro cuya narrativa discuta un tipo de juego, los juegos infantiles, los juegos en el recreo, los juegos en la calle, los juegos y juguetes de un país o una simple actividad recreativa.

Danza. Esta categoría incluye las actividades que relacionan el movimiento con la música, el espacio, el arte y la estética. Los libros que discuten temas como las danzas tradicionales, el ballet, la danza folclórica, la samba, las coreografías, los espectáculos, los bailes de salón, el flamenco u otros, además de la explicación sobre la enseñanza, las rutinas, las dificultades y los distintos movimientos asociados a un tipo de danza, serán incluidos en esta clase.

\section{Naturaleza transversal}

Ética. No se debe confundir ética con educación para la paz. La ética es una categoría más amplia, que incluye nociones de lealtad a un equipo y a los compañeros, otredad, dilemas morales, saber perder y ganar, no hacer trampas en una competición deportiva, el rechazo a las sustancias prohibidas (dopaje), role playing, role model u otras relacionadas con los valores éticos. Los libros que discuten en su narrativa estos temas serán clasificados en esta categoría.

Educación para la paz. Esta categoría está relacionada específicamente con el concepto de paz y de no-violencia. Todos los libros infantiles que utilicen su narrativa para discutir cómo las manifestaciones deportivas pueden contribuir a la prevención de la violencia explícita o implícita (bullying, por ejemplo) o a discutir y fomentar la paz y prevenir o eliminar el problema de la guerra en la sociedad serán incluidos en esta categoría.

Educación ambiental. Esta categoría tiene que ver con la idea de desarrollo sostenible (satisfacer las necesidades del presente sin comprometer las necesidades de las generaciones futuras). Su relación con la narrativa de las obras infantiles se da a través de tres ejes: a) las activida- des deportivas y de ocio pueden agredir el medio ambiente; b) el medio ambiente puede afectar la práctica de una actividad deportiva y de ocio o de un ejercicio físico; c) las actividades deportivas y de ocio pueden proteger el medio ambiente.

Género. La idea clave de este tópico es la coeducación. Una narrativa infantil que aborda un ejercicio físico, un juego o un deporte bajo la perspectiva de la igualdad de oportunidades de ambos sexos será incluida en esta categoría. Un libro que tenga por objetivo discutir el problema del sexismo y, para ello, utilice el deporte es también un ejemplo de esta categoría.

Consumismo. El eje central de esta categoría es comprender e interpretar, a través de la narrativa de los libros infantiles, los mensajes que la sociedad de consumo lanza a todas las personas. Una historia infantil que discuta el consumo, la moda, el marketing, el sponsor y el mercado deportivo deberá ser incluida en esta categoría. Un ejemplo es la gran influencia de las fechas de Navidad y Reyes en el consumismo de juguetes.

Multiculturalismo. La idea clave de esta categoría es discutir la convivencia de diversas culturas en una misma sociedad. Esta pluralidad resulta de la confluencia de tradiciones, culturas, lenguas, creencias, valores y prácticas sociales. En este aspecto en particular, el deporte debe ser visto como un lenguaje universal y como un puente entre las diferencias sociales, étnicas y culturales de los pueblos.

Historia. Un libro que incluya en su narrativa la exposición de acontecimientos pasados, hechos memorables de una persona, un grupo, un equipo o una nación relacionados con la Educación Física y las diferentes manifestaciones deportivas será incluido en esta categoría. La biografía es uno de los ejemplos de esta clase.

Educación gerontológica. Esta categoría tiene relación con el proceso de envejecimiento del ser humano. El hecho de incluir la educación gerontológica en este trabajo se justifica porque el envejecimiento de la población en el siglo XXI es un tema urgente de la agenda académica, política y social de las diferentes áreas de las Ciencias, como las Humanas, las Sociales y las de la Salud. En una visión prospectiva, la literatura indica que este período es el Siglo de las personas mayores. Cualquier libro que discuta en su narrativa nociones de una educación para y sobre el envejecimiento será incluido en este apartado. Algunos ejemplos son las narrativas que abordan el problema del ageism (prejuicio y discriminación) y las actividades intergeneracionales en el deporte.

Fiabilidad del análisis de contenido desarrollado en los libros infantiles

Como bien lo recuerdan Thomas, Nelson y Silverman (2005), «el coeficiente de fiabilidad refleja el grado en el que la medición estálibre de varianza de error» (p. 198).

Por tanto, para validar el análisis de contenido se empleó el criterio de la fiabilidad intraobservador e interobservadores mediante la aplicación del índice kappa de Cohen (1960), que es «un estadístico de concordancia que corrige el azar...» (Bakeman \& Gottman, 1989, p. 109).

Las siguientes etapas explican el proceso de obtención de la fiabilidad del análisis de contenido: 1. ${ }^{\text {a }}$ - determinación del número de los observadores responsables por el análisis (Observadores A y B); 2. ${ }^{\text {a- }}$ utilización de la escala de valoración para el índice kappa de Cohen (1960) propuesta por Landis y Koch (1977, p. 164); 3. ${ }^{\text {- }}$ - cálculo de la fiabilidad intraobservador; 4 . ${ }^{\mathrm{a}}$ - cálculo de la fiabilidad interobsevadores; 5. ${ }^{\text {a- }}$ cálculo de la fiabilidad intraobservador e interobservadores por el programa estadístico.

Los datos de los distintos análisis desarrollados por los Observadores A y B fueron tratados con el software SAS for Windows (XP_PRO Plataform), versión SAS 9.2 TS Level 2MO, con el que se realizó la prueba del índice kappa de Cohen.

El análisis de los enfoques de los libros publicados en Brasil presentó una medida de fiabilidad interobservadores de 0.96 y de fiabilidad intraobservador de 1 (investigador A) y 0.98 (investigador B), mientras que el análisis de los libros publicados en España presentó una medida de fiabilidad interobservadores de 0.98 y de fiabilidad intraobservador de 1 (investigador A) y 0.98 (investigador B). Estos resultados indican 
que los análisis desarrollados tienen una fiabilidad inter e intraobservador casi perfecta (muy buena), según la escala de valoración para el índice kappa de Cohen propuesta por Landis y Koch (1977, p. 165). Esto lleva a la conclusión de que el análisis desarrollado no puede ser considerado subjetivo ni tampoco tendencioso.

\section{Resultados}

\section{Presentación y discusión}

La Tabla 1 hace una yuxtaposición de las frecuencias (absolutas y relativas) de los enfoques identificados en los libros infantiles publicados en Brasil y en España.

\begin{tabular}{lcccc}
\multicolumn{5}{c}{ Tabla 1. Yuxtaposición de los enfoques identificados en los libros infantiles publicados en Brasil y en } \\
España.
\end{tabular}

A continuación, la Figura 3 compara los valores de los enfoques identificados en los libros infantiles publicados en Brasil con los publicados en España.

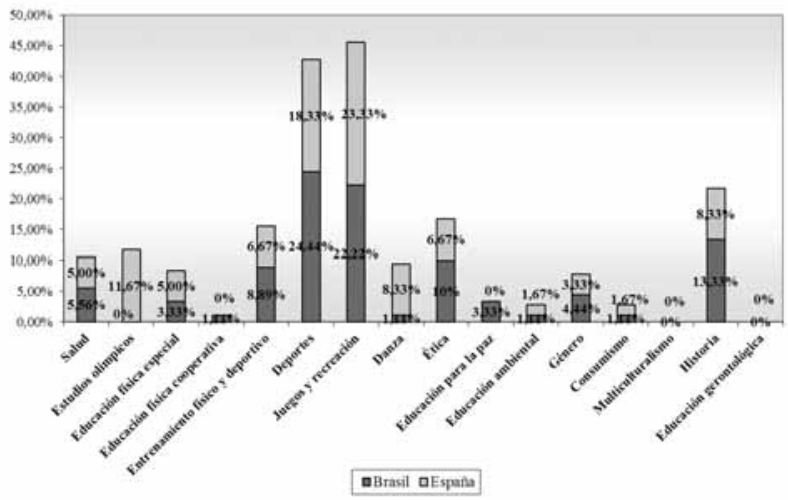

Figura 3. Representación gráfica de la comparación entre los enfoques identificados en los libros infantile publicados en Brasil y en España.

Esta figura demuestra que los enfoques de los Deportes y el de los Juegos y recreación son los que predominan en los dos corpora de libros infantiles. En Brasil, el enfoque de los Deportes (con un 24.44\%) está en primera posición, mientras que en España esta posición la ocupa el enfoque de los Juegos y recreación (con un 23.33\%).

En España, el enfoque de los Estudios olímpicos presenta una significativa incidencia, con un $11.67 \%$, posicionándose en el tercer puesto. Este dato revela que en España, país que organizó la XXV Olimpiada, existe una preocupación por difundir en la literatura infantil las manifestaciones relacionadas con los Juegos Olímpicos. Los libros identificados con este enfoque han sido publicados en 2008, por tanto, tras aproximadamente 16 años de haberse celebrado esa Olimpiada se puede observar la presencia de este tema en la literatura infantil relacionada con la Educación Física y el Deporte.

En cambio, en Brasil no se registra este enfoque en los libros infantiles. Sorprende constatar que ningún libro discuta el enfoque de los Estudios olímpicos, ya que este tema, además de ser un tópico de gran influencia mediática en la sociedad, es de fundamental importancia para el desarrollo del Olimpismo en un país como Brasil, que con su proyecto olímpico ha logrado ser la sede elegida para recibir los Juegos Olímpicos de 2016.

En este aspecto en particular, los profesores e investigadores, así como las editoriales especializadas en Educación Física y Deporte en Brasil, podrían dedicar más atención a la producción, publicación y diseminación de libros infantiles originales con temas relacionados con los Estudios olímpicos.

El enfoque de la Historia presenta una marcada incidencia en ambos países. En Brasil se posiciona en el tercer puesto (con un 13.33\%) y en España está ubicado en la cuarta posición (con un 8.33\%). Este dato demuestra que los autores y autoras de literatura infantil consideran importante abordar, desde el punto de vista de la Historia, las obras con temática relacionada con la Educación Física y el Deporte.

$\mathrm{Al}$ igual que la Historia, otro de los enfoques significativos en los libros publicados en estos dos países es el de la Ética. Los autores que escriben sobre literatura infantil en Brasil y en España consideran que la Ética es un tema propicio para relacionarlo con la Educación Física y el Deporte.

El enfoque de la Salud, que presenta casi los mismos porcentajes en los dos países (en Brasil, un 5.56\%; en España, un 5\%), no alcanza una gran incidencia. Este, sin duda, es un dato interesante, ya que la salud es, históricamente, uno de los temas predominantes en el área de Educación Física.

Respecto al enfoque del Consumismo, los dos países presentan casi los mismos porcentajes.

A su vez, se puede afirmar que apenas hay espacio para el enfoque de la Educación física cooperativa en el ámbito de la literatura infantil publicada en Brasil (incidencia de 1.11\%) y en España el tema no presenta incidencia alguna.

Se aprecia una gran diferencia entre los dos países en cuanto a los libros que difunden temas relacionados con la Danza. En Brasil el tema apenas tiene incidencia (solamente el 1.11\%), mientras que en España presenta una importante incidencia (un 8.33\%).

Otra destacada diferencia entre ambos países es la relacionada con el enfoque de la Educación para la paz. Se considera que éste ha tenido un interesante porcentaje en los libros infantiles publicados en Brasil, incluso sorprendente, ya que dicho enfoque es un tema muy poco abordado en el área de Educación Física en Brasil. En cambio, el tema no ha tenido una única incidencia en las obras infantiles publicadas en España.

Una de las similitudes encontradas es que ambos países no registran incidencia alguna de los enfoques de la Educación gerontológica y del Multiculturalismo.

Con respecto a la Educación gerontológica los autores brasileños y españoles deberían poner más atención al tema, ya que ambos países presentan una alta tasa de envejecimiento de la población (según sus instituciones gubernamentales e institutos nacionales de estadística). La literatura infantil relacionada con la Educación Física y el Deporte puede contribuir a la discusión de temas como el envejecimiento saludable y el ageism.

Con relación al Multiculturalismo, la evidente ausencia de este enfoque en los materiales relacionados con la Educación Física y el Deporte es un problema histórico, reflejando, de esa manera, los referenciales teóricos elegidos por los autores del área en sus investigaciones.

Es un punto bastante negativo la incidencia incipiente del enfoque de la Educación ambiental (el 1.11\%) en las obras infantiles. Como ya mencionado en este texto, existe una estrecha y fuerte relación entre las actividades deportivas y de ocio y el medio ambiente.

\section{Conclusiones y valoración final del análisis comparativo}

La valoración final del análisis comparativo señala que los enfoques de los 150 libros infantiles publicados en Brasil y en España con temática relacionada con la Educación Física y el Deporte reúnen un gran número de similitudes, a saber: 
- Predomina en gran medida el enfoque de los Juegos y recreación en los dos corpora de libros analizados.

- El enfoque de los Deportes también registra una gran incidencia en las obras infantiles de los dos países.

-El enfoque de la Historia presenta una marcada incidencia en ambos p aíses.

- Otro enfoque significativo presente en las obras infantiles analizadas es el de la Ética.

- El enfoque de la Salud, que presenta casi los mismos porcentajes tanto en un país como en otro, no alcanza una gran incidencia.

- Apenas hay espacio para el enfoque de la Educación física cooperativa en el ámbito de la literatura infantil publicada en Brasil (incidencia de 1.11\%) y en España el tema no presenta incidencia alguna.

- Otra de las similitudes encontradas es que ambos países no registran incidencia alguna de los enfoques de la Educación gerontológica y del Multiculturalismo.

Se aprecian tres grandes diferencias entre los dos países, a saber:

- El enfoque de los Estudios olímpicos presenta una significativa incidencia en España, mientras que en Brasil el tema está olvidado.

- El enfoque de la Danza en Brasil apenas tiene incidencia (solamente el 1.11\%), mientras que en España presenta una importante incidencia (un $8.33 \%$ ).

- Se considera que el enfoque de la Educación para la paz ha tenido un interesante porcentaje en los libros infantiles publicados en Brasil, incluso sorprendente, ya que dicho enfoque es un tema muy poco abordado en el área de Educación Física en Brasil. En cambio, el tema no ha tenido una única incidencia en las obras infantiles publicadas en España.

En conclusión, se puede constatar a partir del análisis comparativo aquí desarrollado que los corpora de libros presentan más similitudes que diferencias.

Por ende, se puede considerar que este texto contribuye a dilucidar las similitudes y diferencias de los enfoques de la literatura infantil con temática relacionada con la Educación Física y el Deporte publicada en Brasil y en España.

De una manera prospectiva, se considera que el análisis de libros infantiles con temática relacionada con la Educación Física y el Deporte es un importante paso para la construcción de una base de datos de literatura infantil dirigida a los maestros y maestras de Educación Física en estos dos países.

Es una cuestión necesaria-y también un reto-considerar los libros de literatura infantil con temática relacionada con el Deporte como elementos constitutivos del conjunto de materiales específicos del área deEducación Física.

\section{Referencias}

Bakeman, R., \& Gottman, J. M. (1989). Observación de la interacción: Introducción al análisis secuencial. Madrid: Morata.

Bardin, L. (2002). El análisis de contenido (3. ed.). Madrid: Akal.

Berg, K.E., \& Latin, R.W. (2008). Essentials of research methods in health, physical education, exercise science, and recreation (3rd ed.). Philadelphia: Wolters Kluwer/LippincottWilliams \& Wilkins. Botelho, R.G. (2010). Educación Física y literatura infantil: Posibilidades de utilización en el ámbito escolar (2 v.). (Tesis doctoral no publicada). Departamento de Didáctica de la Expresión Musical, Plástica y Corporal, Facultad de Ciencias de la Educación de la Universidad Autónoma de Barcelona, España. (Incluye el CDROM Banco de libros infantiles de Educación Física y Deporte en España).

Botelho, R.G. (2012). Libros infantiles con temática relacionada con la Educación Física y el Deporte en Brasil: estudio de sus características. Biblios: revista de Bibliotecología y Ciencias de la Información, 49, 44-53. doi: 10.5195/biblios.2012.82

Botelho, R.G, \& Oliveira, C.C. (2011). Estudio sobre el análisis de materia en la literatura infantil relacionada con la Educación Física y el Deporte publicada en España. Biblios: revista de Bibliotecología yCiencias de la Información, 45, 14-27. doi: 10.5195/biblios.2011.35

Cohen, J. (1960). A coefficient of agreement for nominal scales. Educational and Psychological Measurement, 20,37-46.

Delgado, M.A., \& Del Villar, F. (1995). El análisis de contenido en la investigación de la enseñanza de la educación física. Motricidad,1, 25-46.

Egaña, A. (2008). No me gusta el fútbol (3. imp.). (Dibujos de Mikel Valverde, traducción: Juan Cruz Igerabide). Barcelona: La Galera. (¿Y qué? No. 2).

Figueras Adell, N., \& Martorell, E. (2007). El partido del año (Ilustraciones de J. Andrada, traducción P. Rodríguez). Barcelona: La Galera. (El Botiquín de La Galera No. 6).

Fingon, J.C. (2011). Integrating children's books and literacy into the Physical Education curriculum. Strategies: Ajournal for Physical and Sport Educators, 24(4), 10-13.

Heinemann, K. (2008). Introducción a la metodología de la investigación empírica en las ciencias del deporte (2. ed. rev. y ampl.). Badalona: Paidotribo.

Keane, G. (1995). Adão Guaxinim e o valentão Garumf (Tradução: Kátia Mirna Souto Silva). Belo Horizonte: Atos. (Parábolas para crianças).

Landis, J.R., \& Koch, G.G. (1977). The measurement of observer agreement for categorical data. Biometrics, 33, 159-174.

Moraes, F.B., \& Silveira, N. (2002). Natação. Blumenau: Sabida. (Esportes: Brincando de atleta).

Nemer, R.L.P. (2000). O campeão (Ilustrações: Hudson Silva). Rio de Janeiro: Cata-Sonho.

Pirolo, A.L., \& Terra, D.V. (2003). Educación física y deporte escolar: Mismos debates, distintos países (Brasil-España). Retos: Nuevas Tendencias en Educación Física, Deporte y Recreación, 4, 36-41.

Pittar, G. (2004). ¿ Jugamos con el viento? (Ilustrado por Cris Morrell, traducción: Alberto Jiménez Rioja). León: Everest. (Milly Molly).

Thomas, J.R., Nelson, J.K., \& Silverman, S.J. (2005). Research methods in physical activity (5th ed.). Champaign: Human Kinetics.

Weiller, K.H., \& Higgs, C.T. (1989). Female learned helplessness in sport: An analysis of children's literature. Journal of Physical Education, Recreation and Dance, 60(6), 65-67.

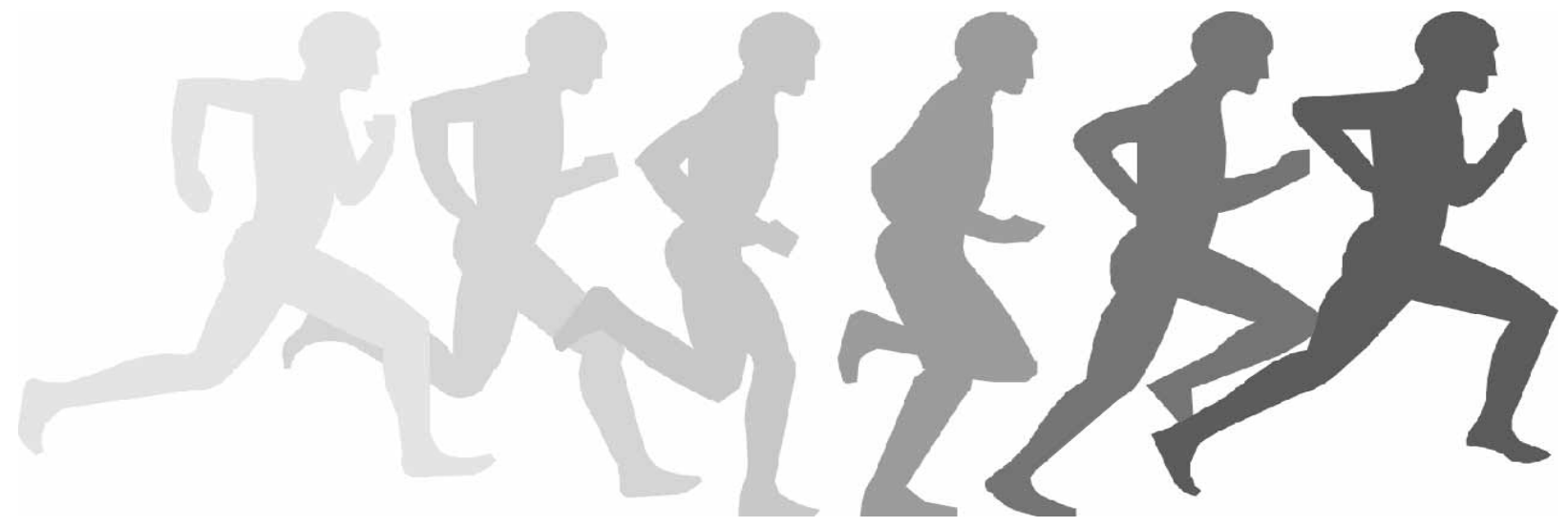

